\title{
Interrogation of Fibre Bragg Grating Sensors Using an Arrayed Waveguide Grating
}

\author{
D C C Norman ${ }^{1}$, D J Webb ${ }^{1}$ and R D Pechstedt ${ }^{2}$ \\ ${ }^{1}$ Photonics Research Group, School of Engineering and Applied \\ Science, Aston University, Aston Triangle, Birmingham, \\ B4 7ET, United Kingdom \\ ${ }^{2}$ Bookham Technology Ltd, 90 Milton Park, Abingdon, \\ Oxfordshire, OX14 4RY, United Kingdom \\ E-mail: d.j.webb@aston.ac.uk
}

Received 19 September 2003, accepted for publication 9 October 2003

Published 4 November 2003

Online at stacks.iop.org/MST/15/148 (DOI: 10.1088/0957-0233/15/1/021)

\begin{abstract}
We experimentally investigate the use of an arrayed waveguide grating (AWG) to interrogate fibre Bragg grating (FBG) sensors. A broadband light source is used to illuminate the FBG sensors. Reflected spectral information is directed to the AWG containing integral photodetectors providing 40 electrical outputs. Three methods are described to interrogate FBG sensors. The first technique makes use of the wavelength dependent transmission profile of an AWG channel passband, giving a usable range of $500 \mu \varepsilon$ and a dynamic strain resolution of $96 \mathrm{n} \varepsilon / \sqrt{\mathrm{Hz}}$ at $13 \mathrm{~Hz}$. The second approach utilises wide gratings larger than the channel spacing of the AWG; by monitoring the intensity present in several neighbouring AWG channels an improved range of $1890 \mu \varepsilon$ was achieved. The third method improves the dynamic range by utilising a heterodyne approach based on interferometric wavelength shift detection providing an improved dynamic strain resolution of $17 \mathrm{n} \varepsilon / \sqrt{ } \mathrm{Hz}$ at $30 \mathrm{~Hz}$.
\end{abstract}

Keywords: Arrayed waveguide grating, fibre Bragg grating, heterodyne, sensor

\section{Introduction}

The basic requirements for fibre Bragg grating (FBG) sensor interrogation systems are high resolution with a large measurement range, multiplexing capability and competitive pricing.

Holographically written fibre Bragg gratings (FBGs) were proposed for use as sensing elements in 1989 [1] since then we have seen continual improvement in the quality and performance of FBGs and FBG sensor interrogation schemes. Charge-coupled device arrays [2], adaptive filters [3], acousto-optic tuneable filters [4] and semiconductor optical amplifiers [5] have all been successfully demonstrated to interrogate FBG sensor systems.

To achieve quasi-distributed sensing, FBGs can be written into a length of fibre and addressed using wavelength division multiplexing (WDM) [6]. FBG sensors can be easily embedded into a variety of materials, e.g. to monitor the structural heath of bridges [7].

The advantages of using fibre optic sensors over their electro-mechanical counterparts include their ability to be small in size, their resistance to electromagnetic interference, high sensitivity and high bandwidth [8]. FBGs are considered excellent sensor elements, suitable for measuring static and dynamic fields such as strain, temperature, pressure and magnetic fields [9-12].

Phased array multiplexers or arrayed waveguide gratings (AWGs) were first proposed in 1988 [13]. The first long wavelength AWGs appeared in the early 1990s [14, 15] and were seen as key components in the development of WDM communications systems, which 
required multiplexers and demultiplexers to combine signals from several sources over a single fibre. By utilizing "off the shelf" optical devices such as an arrayed waveguide grating (AWG) we envisage the overall costs of FBG systems will ultimately be reduced helping to displace their electro-mechanical counterparts.

In this paper we report on a detailed investigation of the use of an AWG as part of an interrogation system for FBG sensors $[16,17]$. In all the schemes described, the AWG acts as a wavelength demultiplexer, separating out the signals from individual FBGs; in some of the schemes it is also used to recover the measurand induced wavelength shift. We demonstrate extended range interrogation using a wide-bandwidth grating approach and a heterodyne approach based on interferometric wavelength shift detection.

\section{AWG Operation}

A schematic of an AWG configured as a demultiplexer is shown in figure 1 . The AWG demultiplexer consists of two planar slab waveguides with an array of waveguides connecting them. When broadband light enters the first slab waveguide it is no longer laterally confined but diffracts into the waveguide array. The arrayed waveguide is designed such that the optical path length difference (OPD) between adjacent waveguides ensures light emerges with different and wavelength dependent relative phases. Thus constructive interference occurs in different directions for different wavelengths, allowing spatial separation of different wavelength channels to be achieved [18]. The channel wavelengths are usually matched to the channels specified by the international telecommunications union and display typical channel separations of 25,50 or $100 \mathrm{GHz}$ (roughly $0.2,0.4$ or $0.8 \mathrm{~nm}$ in the C-Band). The experimental work reported in this paper was carried out using an Optical Channel Monitor (OCM) from Bookham Technology. The Bookham OCM is a solid-state, thermally stabilised 40 channel AWG with integral photodetectors that provides 40 electrical outputs. The voltage level present at each electrical output represents the power level in each AWG channel. The OCM is designed with a channel spacing of $100 \mathrm{GHz}$ or $0.8 \mathrm{~nm}$ and a $1 \mathrm{~dB}$ passband per channel of $\pm 100 \mathrm{pm}$.

\section{Experimental Arrangement}

The experimental set-up for interrogating FBG sensors using an AWG is shown in figure 2. A non-polarised erbium-doped fibre amplified spontaneous emission source provided a total output power of $12 \mathrm{dBm}$ over a spectral width at $-3 \mathrm{~dB}$ of $33 \mathrm{~nm}$ centred about a wavelength of $1545 \mathrm{~nm}$. The light passed through an unbalanced Mach-Zehnder interferometer. One arm contained an integrated-optic phase modulator, to which a ramp signal could be applied. The OPD in the other arm could be adjusted and also blocked to effectively remove the interferometer. Polarisation controllers were used in both arms of the interferometer to optimise the fringe visibility at the output of the interferometer. The light then passed through a coupler arrangement, which directed the light to an optical spectrum analyser to monitor the interferometer output, and selectively to the fibre grating sensors. The fibre grating sensors consisted of a chirped Bragg grating mounted on a fibre stretcher and two FBG sensors one of which was mounted on a second fibre stretcher with an integral piezoelectric transducer (PZT) stretcher so that dynamic strain signals could be applied. The second FBG (FBG 2) had a wavelength several nanometers from the first so that light reflected from it appeared in an AWG channel some distance away from FBG 1. The reflected light from one of the two sensor fibres was passed to the OCM to which could be connected the relevant electronic interrogation system.

\section{FBG Sensing}

The simplest technique to interrogate multiplexed FBG sensors makes use of the AWG passband profile. If the nominal wavelength of an FBG lies within an AWG passband any variation in the FBG wavelength will produce a related variation in the detected output intensity. This principle is shown in figure 3. To show this effect experimentally we blocked one arm of the interferometer allowing the broadband light to be directed to the PZT mounted FBG (FBG 1). An FBG was selected with a centre wavelength of $1551 \mathrm{~nm}$ and a reflected $-3 \mathrm{~dB}$ linewidth of $0.48 \mathrm{~nm}$. The strain induced wavelength change shifts the reflected power further into the passband of channel 13 through it and into channels 12 and 11. Figure 4 shows the change in voltage level at the corresponding electrical outputs of the OCM. The results indicate that good sensitivity, which is obtained in the steepest part of the AWG channel passband, limits the usable range to less than $0.5 \mathrm{~nm}$. Homodyne dynamic tests were carried out by applying a sinusoidal modulation of $15 \mu \varepsilon$ at $13 \mathrm{~Hz}$ to the same FBG via the PZT. The grating was stretched to bring its wavelength to the edge of the steepest part of the corresponding AWG channel passband to produce the best signal to noise ratio (SNR). The spectrum of the observed signal is shown in figure 5 . The peaks in order of increasing frequency are the signal at $13 \mathrm{~Hz}$, its first and third harmonics and $50 \mathrm{~Hz}$ mains voltage interference. With a SNR of $50 \mathrm{~dB}$, the maximum dynamic strain resolution obtained was $96 \mathrm{ne} / \sqrt{ } \mathrm{Hz}$.

This simple approach, which has been previously described [13], suffers from two main problems: firstly that being essentially intensity based the output is also influenced by changing losses in the system, and secondly the useable range is limited to the rising part of the channel passband, less than $0.5 \mathrm{~nm}$ equivalent to around $500 \mu \varepsilon$. To overcome these limitations we have investigated an approach using a wide-bandwidth grating scanned over several channels of the AWG as 
well as a heterodyne approach using interferometric wavelength shift detection [19].

\subsection{Wide-bandwidth grating approach}

This approach involves the use of wide gratings that span a few AWG channels. To facilitate this we manufactured a chirped grating using the phase mask technique [20, 21]. This provided us with a grating centred on $1548.16 \mathrm{~nm}$ with a $-3 \mathrm{~dB}$ bandwidth of $5.4 \mathrm{~nm}$. With one arm of the interferometer still blocked the reflected light from the chirped grating mounted on the fibre stretcher was directed to the AWG. The reflected power of the grating appeared in channels 11 $(1552.52 \mathrm{~nm})$ to $21(1544.53 \mathrm{~nm})$. An analogue to digital converter and a personnel computer (PC) were connected to the corresponding OCM outputs and a few more up to channel $6(1556.55 \mathrm{~nm})$ so the reflected power from the grating as it was stretched could be recorded. Figure 6 shows the response in the absence of any strain. To recover the grating position we used centroid detection, given by [7]

$$
\text { Centroid }=\frac{\sum\left(i \times V_{i}\right)}{\sum V_{i}}
$$

Where, $i$ is the OCM channel sampled and $\mathrm{V}_{\mathrm{i}}$ is the $i^{\text {th }}$ OCM channel voltage output.

We started by stretching the grating consecutively by $10 \mu \mathrm{m}$ (or $54 \mu \varepsilon$ ) calculating and plotting the centroid position using equation (1); the results are shown in figure 7 . The results show that a near linear response is achieved from the centroid fit of the data displaying a range of $1890 \mu \varepsilon$ corresponding to the grating being stretched through only two channels of the AWG or $1.6 \mathrm{~nm}$. The range is a considerable improvement over the simple FBG interrogation technique mentioned previously. The resolution for these quai-static measurements, defined as the rms deviation from linearity, is $1.29 \mu \varepsilon$.

\subsection{Heterodyne approach}

With this approach we reintroduced the fibre MachZehnder interferometer and modulated its phase using a $2 \pi$ amplitude serrodyne waveform. The FBGs (FBG 1 and FBG 2) reflect a portion of the interferometer output swept over time, which produces a sinusoidally oscillating intensity $I(\lambda)$ back-reflected from the gratings, given by $[19,22]$

$$
I(\lambda)=A(1+k \cos (\psi(\lambda)+\phi))
$$

Where, A is proportional to the input intensity and system losses, $\mathrm{k}$ is the visibility of the interference signal, the phase term $\Psi(\lambda)=2 \pi \mathrm{OPD} / \lambda$ and $\phi$ is a phase bias drift term that accounts for random length variations in the arms of the interferometer. After photo-detection this gives an electrical carrier, phase modulated by any measurand-induced wavelength shift from the FBG sensors. For a fixed OPD in the Mach Zehnder interferometer, the interferometer therefore serves as a wavelength discriminator. The relationship between a shift in the FBG wavelength $\lambda_{\mathrm{B}}$ and the interferometer phase $\delta \Psi$ is given by [19]

$$
\delta \psi=\frac{-2 \pi O P D}{\lambda_{B}^{2}} \delta \lambda_{B}
$$

The strain induced phase change $\delta \Psi$ can now be detected by monitoring the sensor carrier phase shift relative to the fixed phase of the serrodyne modulation, or alternatively by using FBG 2 as an isolated reference grating largely cancelling the effects of the random phase bias drift $\phi[19]$.

To extend the range of the demodulation scheme using an AWG we simply electrically sum the outputs from several adjacent OCM channels. The issue then becomes how the signal to noise ratio varies, particularly when the FBG is situated between two passbands. To investigate this dependence, a low frequency strain amplitude of $1.5 \mu \varepsilon$ at $30 \mathrm{~Hz}$ was applied to four different FBGs mounted successively on the combined fibre stretcher and PZT. Three of the FBG's were manufactured with increasing bandwidths of $0.13,0.46$ and $0.63 \mathrm{~nm}$ and a fourth was produced with a double peaked structure using the phase mask technique [20], the fourth grating being stretched mid exposure to produce the difference in wavelength between the two peaks $[23,24]$. A $2 \pi$ serrodyne modulation was applied at a frequency of $10 \mathrm{kHz}$ using the phase modulator to create a carrier. The first FBG under test had a strain-free centre wavelength of $1558.29 \mathrm{~nm}$ and a $-3 \mathrm{~dB}$ linewidth of $0.13 \mathrm{~nm}$. The free spectral range (FSR) of the interferometer was $0.8 \mathrm{~nm}$ at the wavelength of FBG 1.

A typical recovered signal when the FBG was in the centre of an AWG passband is shown in figure 8. The data in figure 8 demonstrate a noise limited strain amplitude resolution of around $17 \mathrm{ne} / \sqrt{\mathrm{Hz}}$, which is significantly better than that obtained with the homodyne approach described earlier. By stretching the first FBG through a few channels of the AWG the signal to noise ratio of a sideband as a function of the centre wavelength of the FBG was recorded. Figure 9 shows a plot of the SNR in $\mathrm{dB}$ as the centre wavelength of the FBG passes through channels 3,2 and a small part of channel 1. As expected, the best SNR lies at the centre of each channel. We then added the 3 channels together electrically, the plot of which is also shown in figure 9. This yielded a comparable centre-of-band signal level with a small $(5 \mathrm{~dB})$ increase at the channel crossovers compared to the individual channels. The noise (variation in the signal level) visible on these and subsequent traces is predominantly due to the use of the fibre Mach-Zehnder interferometer. The interferometer is prone to low frequency fluctuations due to draughts or temperature changes in the laboratory. This is not a 
fundamental problem though as the effects would be much reduced were an integrated optic interferometer to be used instead.

It must be stressed that the fall in SNR mid-channel does not imply a drop in the sensitivity (phase change per unit strain) of the demodulation. This is because the carrier amplitude drops in the same way and it is the ratio of sideband to carrier amplitudes that determines the level of phase modulation. The drop in SNR does however imply a worsening in the noise-limited resolution in this region by about a factor of ten compared to the centre-band case.

The second grating had a centre wavelength of $1551.29 \mathrm{~nm}$ with a $3 \mathrm{~dB}$ linewidth of $0.46 \mathrm{~nm}$. The strain amplitude of $1.5 \mu \varepsilon$ at $30 \mathrm{~Hz}$ was applied and the FBG stretched through the corresponding AWG channels, this time channel 13 to channel 11. Figure 10 shows the separate channel signal to noise ratios from this grating along with the summed output. Because the FBG's linewidth is wider than the previous grating the channel crossover points have a higher signal level, due to the fact that more of the reflected light is present in adjacent channels. When the channels are added together the resultant signal level is relatively constant, with in fact a slight increase in the signal level at the crossovers due to this contribution of adjacent channel powers.

The third grating had a centre wavelength of $1550.95 \mathrm{~nm}$ with a $3 \mathrm{~dB}$ linewidth of $0.63 \mathrm{~nm}$. The results for this grating are shown in figure 11. The slightly wider linewidth of this FBG closes the gap between adjacent channels even more, resulting in a further increase in SNR at the channel crossovers. The gradual overall increase in SNR with wavelength is due to the non-uniform source spectrum. We tested this FBG to destruction to see how far the range could be extended. The response continued over a range of about 4.7nm (nearly $5 \mathrm{~m} \varepsilon$ ) before the fibre broke.

From the above results it is clear that the use of wider gratings allows the recovery of an approximately constant signal level as the FBG sweeps through the AWG channels. However, the width of the FBG ultimately places a limit on the sensitivity available. With interferometric wavelength shift detection, the sensitivity is improved by increasing the interferometer OPD and thereby reducing the FSR of the interferometer, but the FSR cannot be made smaller than the width of the FBG being interrogated without significant carrier amplitude reduction; this places an upper limit on the sensitivity, which may be problematic if wide bandwidth FBGs are used.

To try to overcome this problem the final grating tested was grown with double peaks one at $1549.78 \mathrm{~nm}$ with a $3 \mathrm{~dB}$ bandwidth of $0.14 \mathrm{~nm}$ and the other at $1550.98 \mathrm{~nm}$ with a $3 \mathrm{~dB}$ bandwidth of $0.12 \mathrm{~nm}$, the peakto-peak difference of $1.2 \mathrm{~nm}$ corresponding to one and a half channel spacings of the AWG. The idea with this approach is that when one peak is in-between two channels and hence returning a weak signal, the other peak is centred on another channel and returning a strong signal. The OPD of the interferometer was adjusted so that the FSR was equal to $0.6 \mathrm{~nm}$ enabling in-phase signals to be recovered from each peak. So long as the FSR of the interferometer is an integral submultiple of the FBG peak spacing, the two signals will be in phase and add constructively. The recovered response is shown in figure 12 . It can be seen that there is very little variation in SNR across the channels. For the double peaked FBG an average noise limited resolution of $17 \mathrm{n} \varepsilon / \sqrt{ } \mathrm{Hz}$ was obtained.

\section{Conclusion}

The experimental work reported has demonstrated that we can use AWGs to successfully interrogate fibre Bragg grating sensors. The static range of FBG sensor interrogation was improved using a chirped grating spanning several channels of the AWG and detecting the grating position using a centroid fit of the recovered data. We can select the range of the wide-bandwidth approach by simply sampling the appropriate OCM channels with the PC. The dynamic range was also improved by implementing a heterodyne approach based on interferometric wavelength shift detection. By electrically adding channels of the OCM the range of the strain induced wavelength shift can be extended indefinitely. The most uniform response was obtained with a wide grating bandwidth or the double peak structure. While double peaked FBG profiles are slightly more complicated to record, they offer the possibility of increasing the sensitivity because the two peaks can be made with narrow bandwidths.

The advantages of using the OCM arrangement is clearly in the ease of processing the voltage outputs in the analogue or digital domain.

\section{References}

[1] Meltz G, Morey W W and Glenn W H 1989 Formation of Bragg gratings in optical fibers by a transverse holographic method Opt. Lett. 14 823-825

[2] Ezbiri A, Kanellopoulos S E and Handerek V A 1998 High resolution instrumentation systems for fibre Bragg grating aerospace sensors $O p t$. Commun. 150 43-48

[3] Russel J J, Davis M A, Sirkis J, Kersey A D and Lara H 2003 Adaptive-filter based Bragg grating demodulator $16^{\text {th }}$ Int. Conf on Optical Fibre Sensors (OFS-16, Nara, Japan) Tech. Digest 538-541

[4] Xu M G, Geiger H, Archambault J L, Reekie L and Dakin J P 1993 Novel interrogating system for fibre Bragg grating sensors using an acoustooptic tuneable filter Elec. Lett. 29 1510-1511

[5] Lloyd G D, Everall L A, Sugden K and Bennion I 2004 A high-performance miniaturized time division multiplexed sensor system for remote structural heath monitoring (Conf on Optical 
Sensing, Photonics Europe. Strasbourg, France) Proc. SPIE. 5459

[6] Rao Y J 1997 In-fibre Bragg grating sensors, Meas. Sci. Technol. 8 355-375

[7] Calvert G S, Conte P J, Moaveni B, Schulz L W and Callafon R 2003 Structural damage detection using fibre Bragg gratings and modal analysis on a carbon shell bridge girder $16^{\text {th }}$ Int. Conf on Optical Fibre Sensors (OFS-16, Nara, Japan) Tech. Digest 478-481

[8] Yu F T S and Yin S 2002 Fiber Optic Sensors (Marcel Dekker) 1-2

[9] Othonos A and Kalli K 1999 Fiber Bragg gratings: Fundamentals and applications in telecommunications and sensing (Artech House) 301-388

[10] Morey W W, Meltz G and Glenn W H 1989 Fibre optic Bragg grating sensors Proc. SPIE. 1169 98-107

[11] Morey W W, Meltz G and Welss J M 1992 Evaluation of a fibre Bragg grating hydrostatic pressure sensor Proc. $8^{\text {th }}$ Int. Conf. On Optical Fibre Sensors (Monterey, CA, USA) postdeadline paper: $\mathrm{PD}-4$

[12] Kersey A D and Marrone M J 1994 Fibre Bragg grating high-magnetic-field probe $199410^{\text {th }}$ Int. Conf. On Optical Fibre Sensors (Glasgow, UK). Proc. SPIE. 2360 53-56

[13] Smit M K 1988 New Focusing and Dispersive Planar Component Based on an Optical Phased Array Elec. Lett. 24 385-386

[14] Takahasi H, Suzuki S, Kato K and Nishi I 1990 Arrayed-Waveguide Grating for Wavelength Division Multi/Demultiplexer with Nanometer Resolution Elec. Lett. 26 87-88

[15] Takahasi H, Nishi I and Hibino Y 1992 10Ghz Spacing Optical Frequency Division Multiplexer Based on Arrayed-Waveguide Grating Elec. Lett. 28 380-382

[16] Sano Y, Hirayama N and Yoshino T 2003 Fast Optical Wavelength Interrogator Employing Arrayed Waveguide Grating for Distributed Fiber Bragg Grating Sensors IEEE J. Lightwave Technol. 21 132-139

[17] Berkoff T A and Kersey A D 1996 Fibre Bragg grating array sensor system using a bandpass wavelength division multiplexer and interferometric detection IEEE Photon. Technol. Lett. 8 1522-152

[18] Smit M K and Van Dam C 1996 PHASAR-Based WDM devices: Principles, design and applications IEEE J. Topics Quantum Elec 2 236-250
[19] Kersey A D, Berkoff T A and Morey W W 1992 High-Resolution Fibre-Grating Based Strain sensor with Interferometric Wavelength-Shift Detection Elec. Lett. 28 236-238

[20] Hill K O, Malo B, Bilodeau F, Johnson D C and Albert J 1993 Bragg gratings fabricated in monomode photosensitive optical fiber by UV exposure through a phase mask App. Phys. Lett. 62 1035-1037

[21] Kashyap R, McKee P F, Campbell R J and Williams D L 1994 Novel method of producing all fibre photoinduced chirped gratings Elec. Lett. 30 996-998

[22] Kersey A D, Berkoff T A and Morey W W 1992 High-resolution fibre-grating based strain sensor with interferometric wavelength-shift detection Elec. Lett. 28 236-238

[23] Campbell R J and Kashyap R 1995 Spectral profile and multiplexing of Bragg gratings in photosensitive fibre Opt. Lett. 16 898-900

[24] Zhang Q, Brown D A, Reinhart L, Morse T F, Wang J Q and Xiao G 1994 Tuning Bragg wavelength by writing gratings on prestrained fibres IEEE Photon. Tech. Lett. 6 839-841 


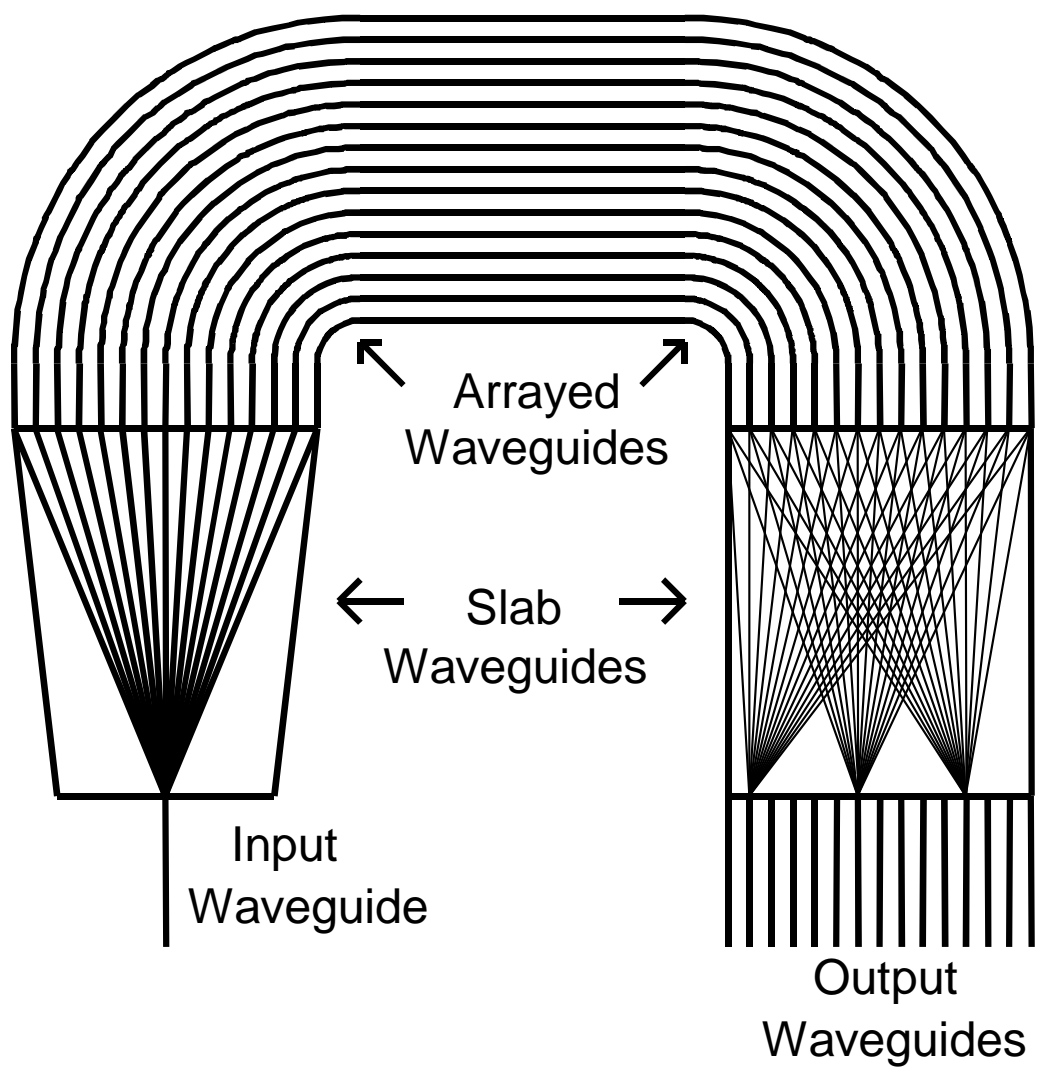

Fig. 1. Schematic layout of an arrayed waveguide grating demultiplexer. 


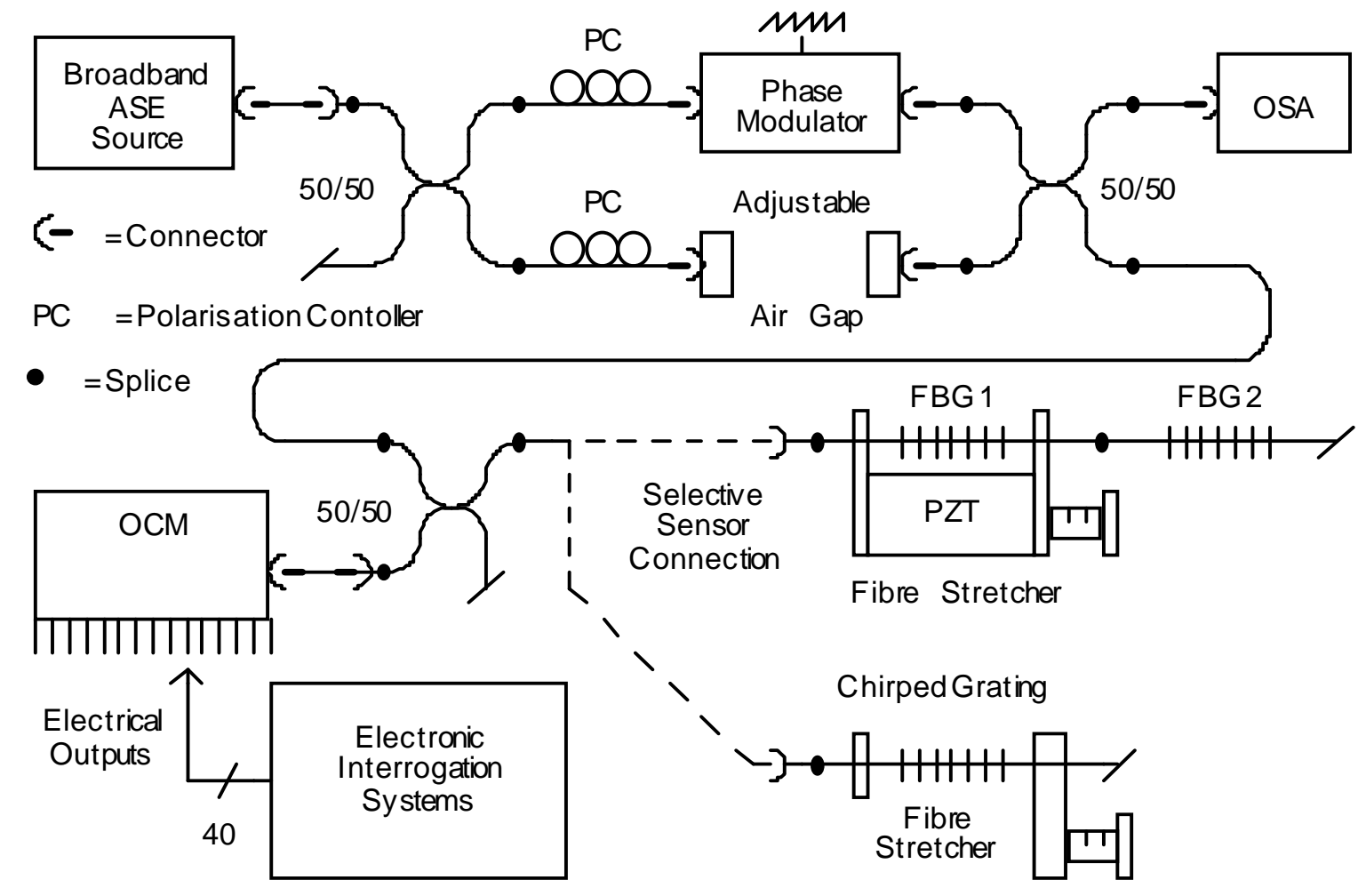

Fig. 2. Experimental set-up. 


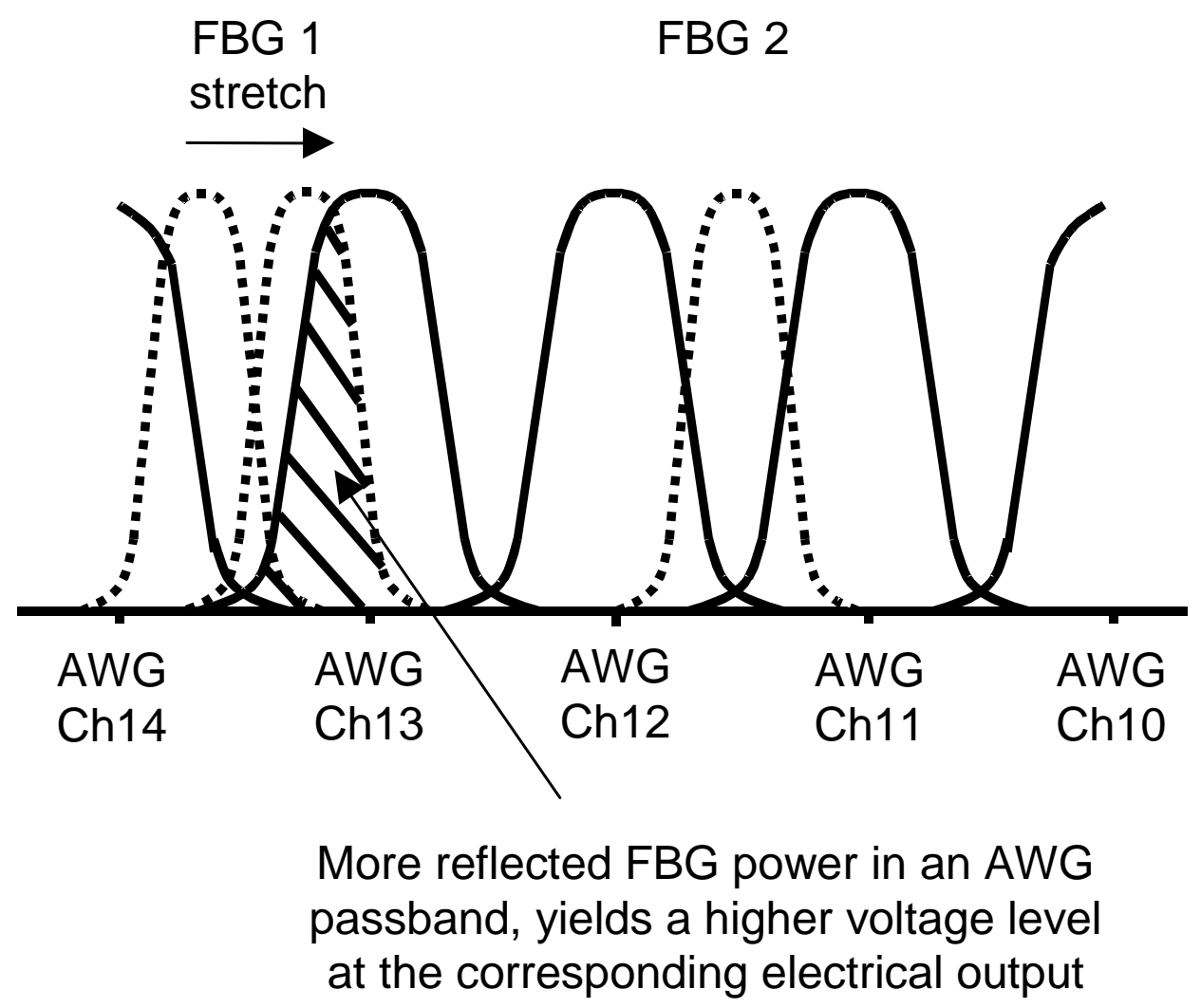

Fig. 3. Principle of straining an FBG into an AWG channel. 


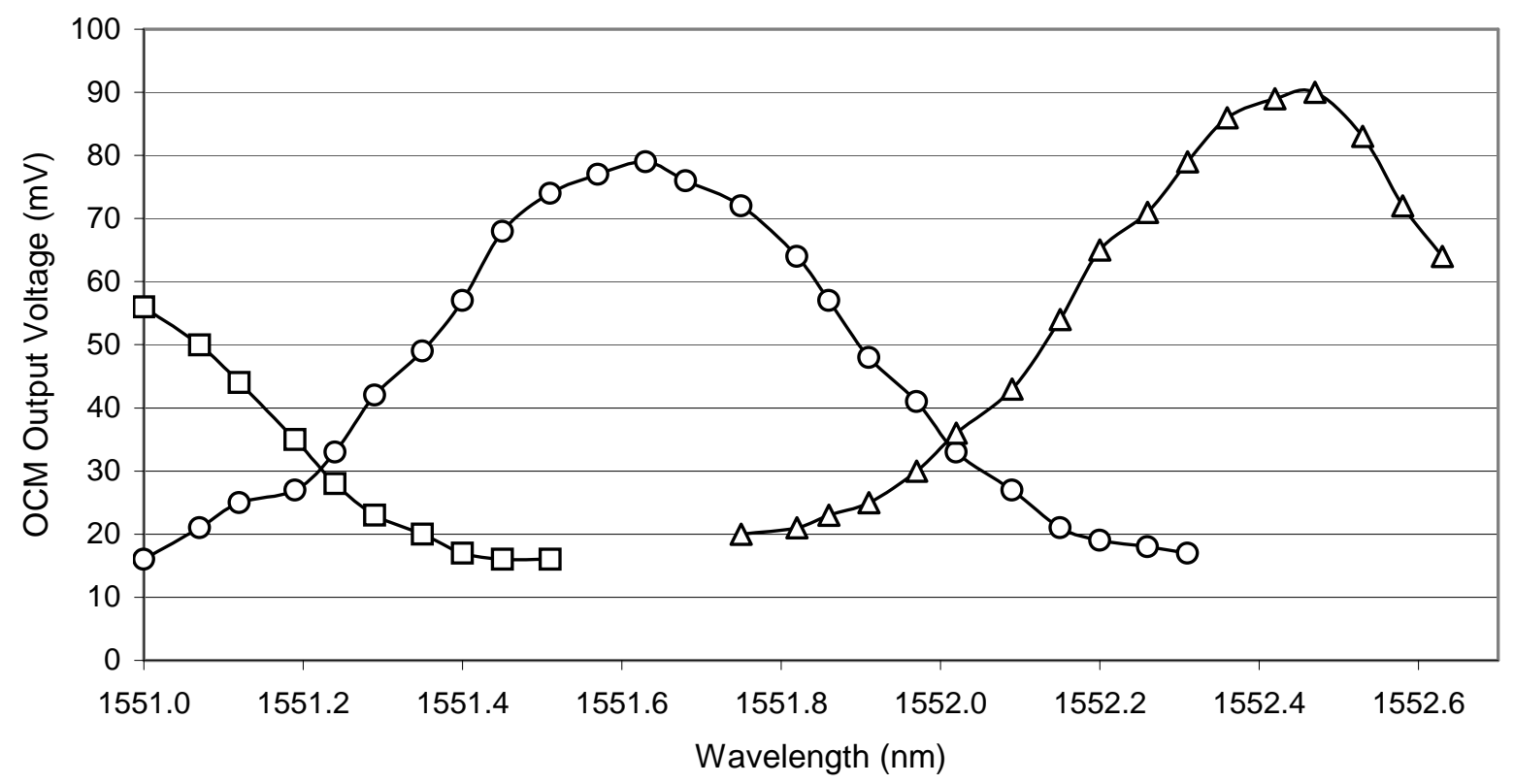

Fig. 4. Change in the OCM output voltage as an FBG is stretched through channels 13 (squares), channel 12 (circles) and channel 11(triangles). 


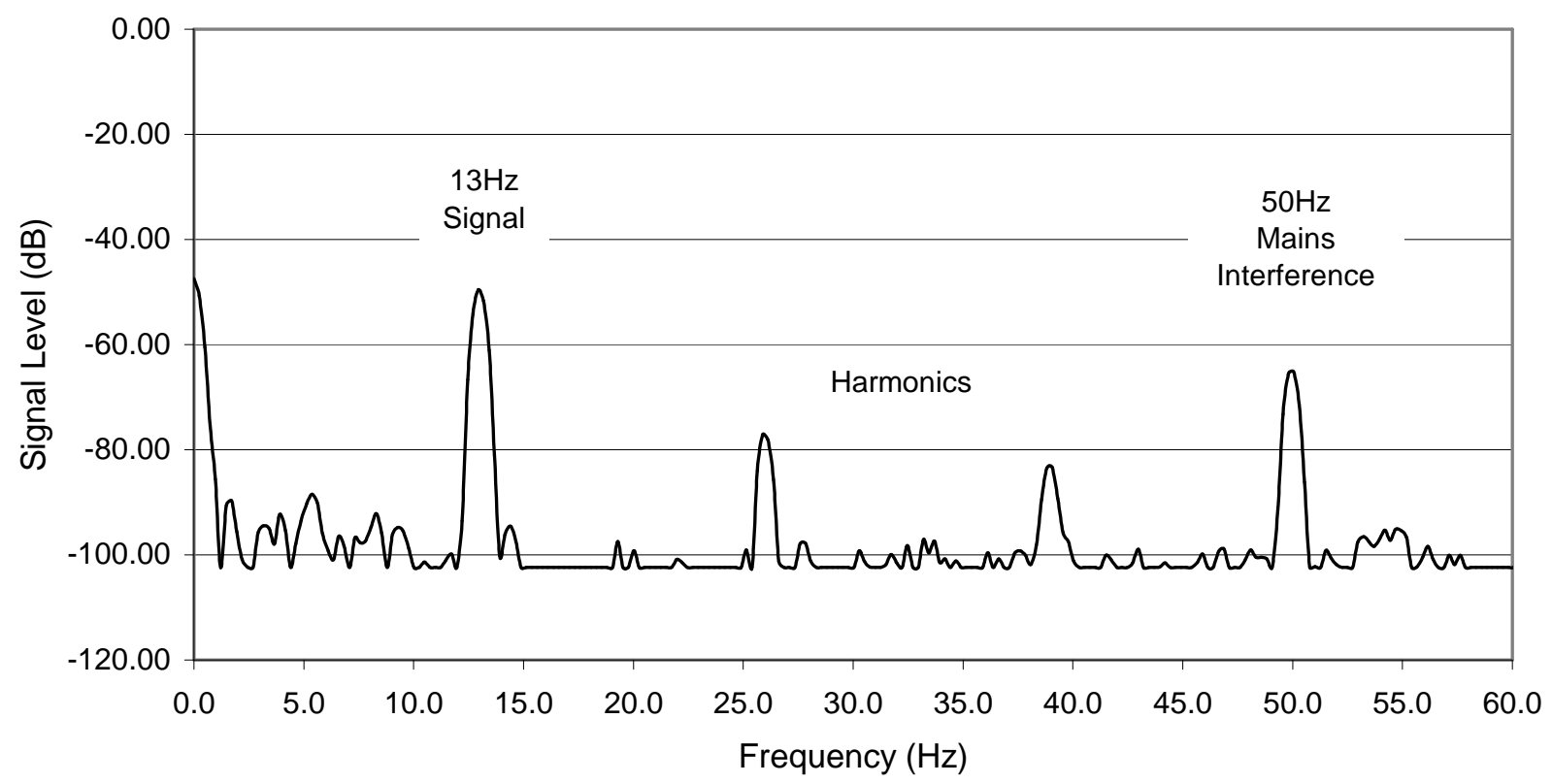

Fig. 5. Homodyne test results due to a FBG with an applied sinusoidal modulation of $15 \mu \varepsilon$ at $13 \mathrm{~Hz}(244 \mathrm{mHz}$ bandwidth). 


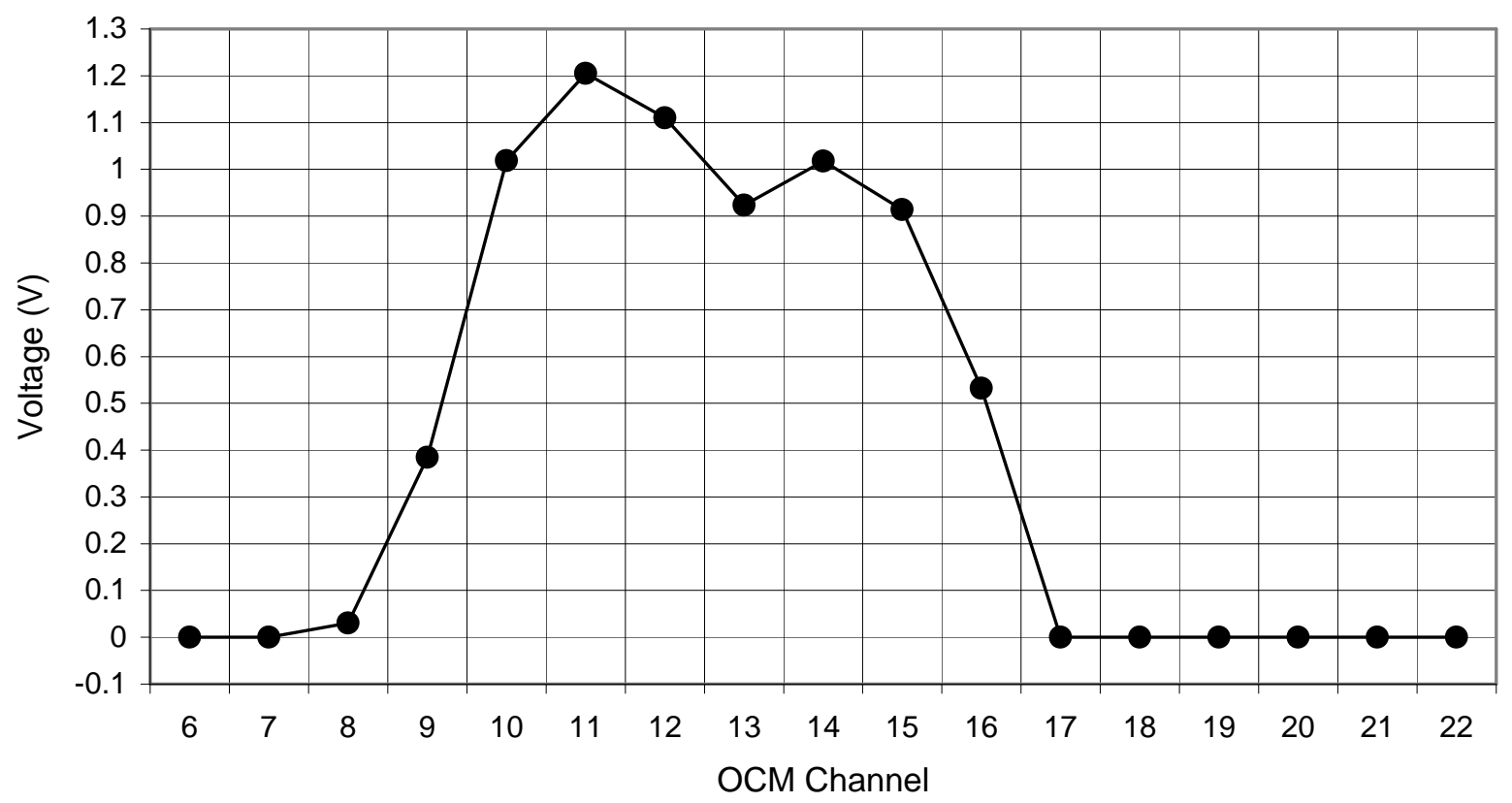

Fig. 6. Reflected chirped grating response as seen on a PC. 


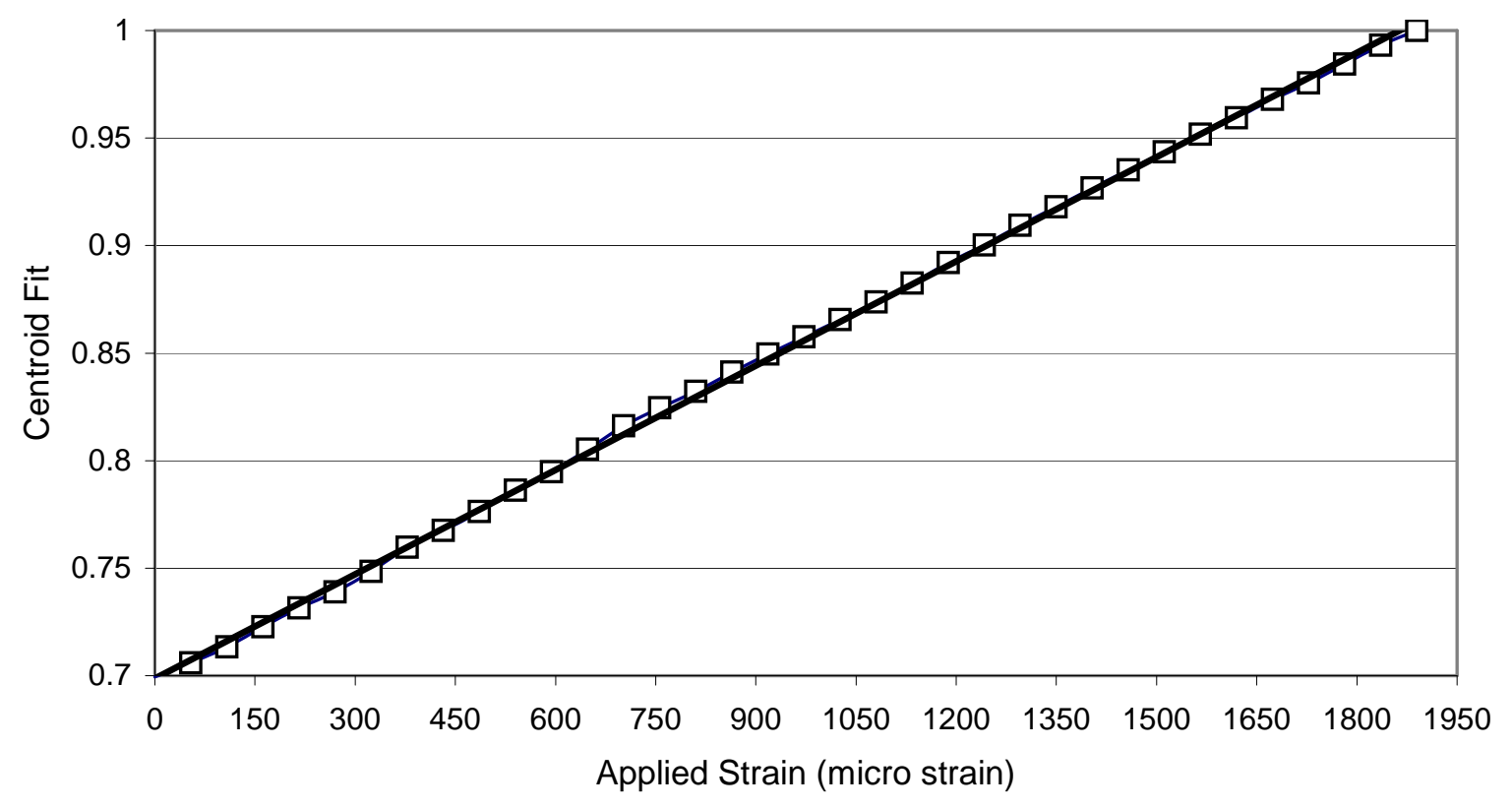

Fig. 7. Centroid fit vs. applied strain (squares). Least squares fit to the data indicated by solid line (resolution $1.29 \mu \varepsilon$ ). 


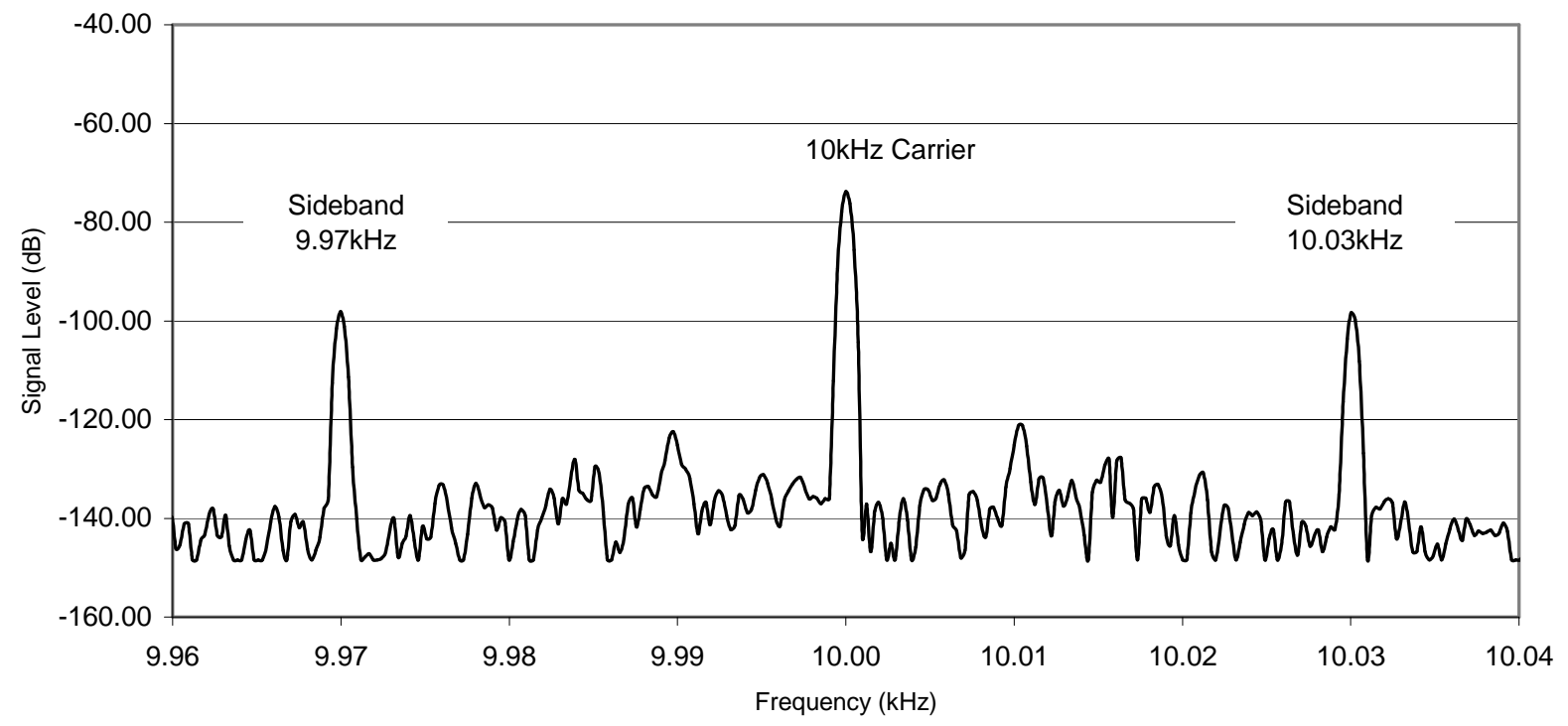

Fig. 8. Typical spectrum due to a FBG with applied sinusoidal modulation of $1.5 \mu \varepsilon$ at $30 \mathrm{~Hz}$ on a $10 \mathrm{kHz}$ carrier $(244 \mathrm{mHz}$ bandwidth). 


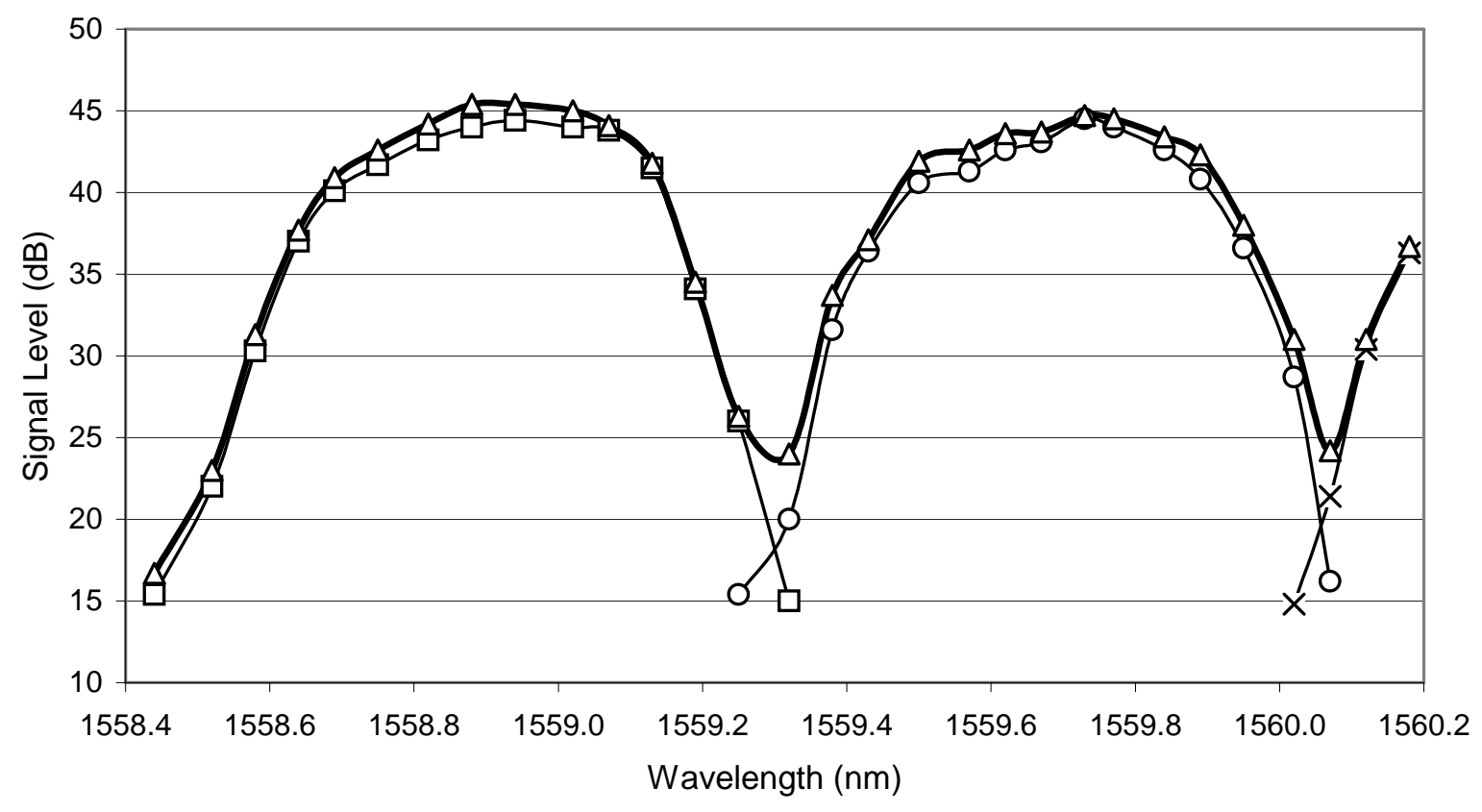

Fig. 9. Signal to noise ratio vs. wavelength for the first FBG stretched through channel 3 to channel 1 with a strain amplitude of $1.5 \mu \varepsilon$ at $30 \mathrm{~Hz}$. Channel 3 (squares), channel 2 (circles), channel 1 (crosses) and channels $3+2+1$ (triangles). 


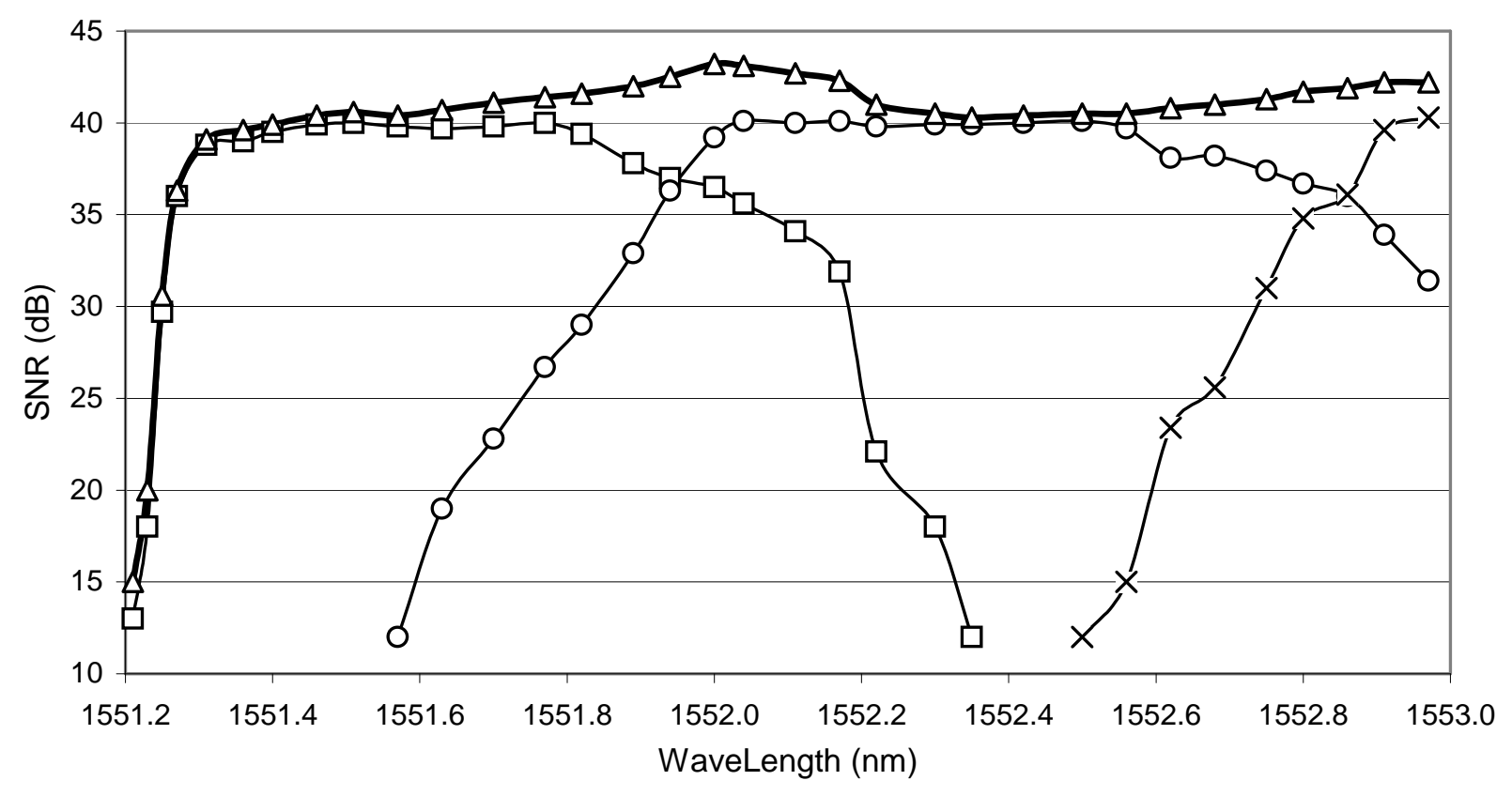

Fig. 10. Signal to noise ratio vs. wavelength for the second FBG stretched through channel 12 to channel 10 with a strain amplitude of $1.5 \mu \varepsilon$ at $30 \mathrm{~Hz}$. Channel 12 (squares), channel 11 (circles), channel 10 (crosses) and channels $12+11+10$ (triangles). 


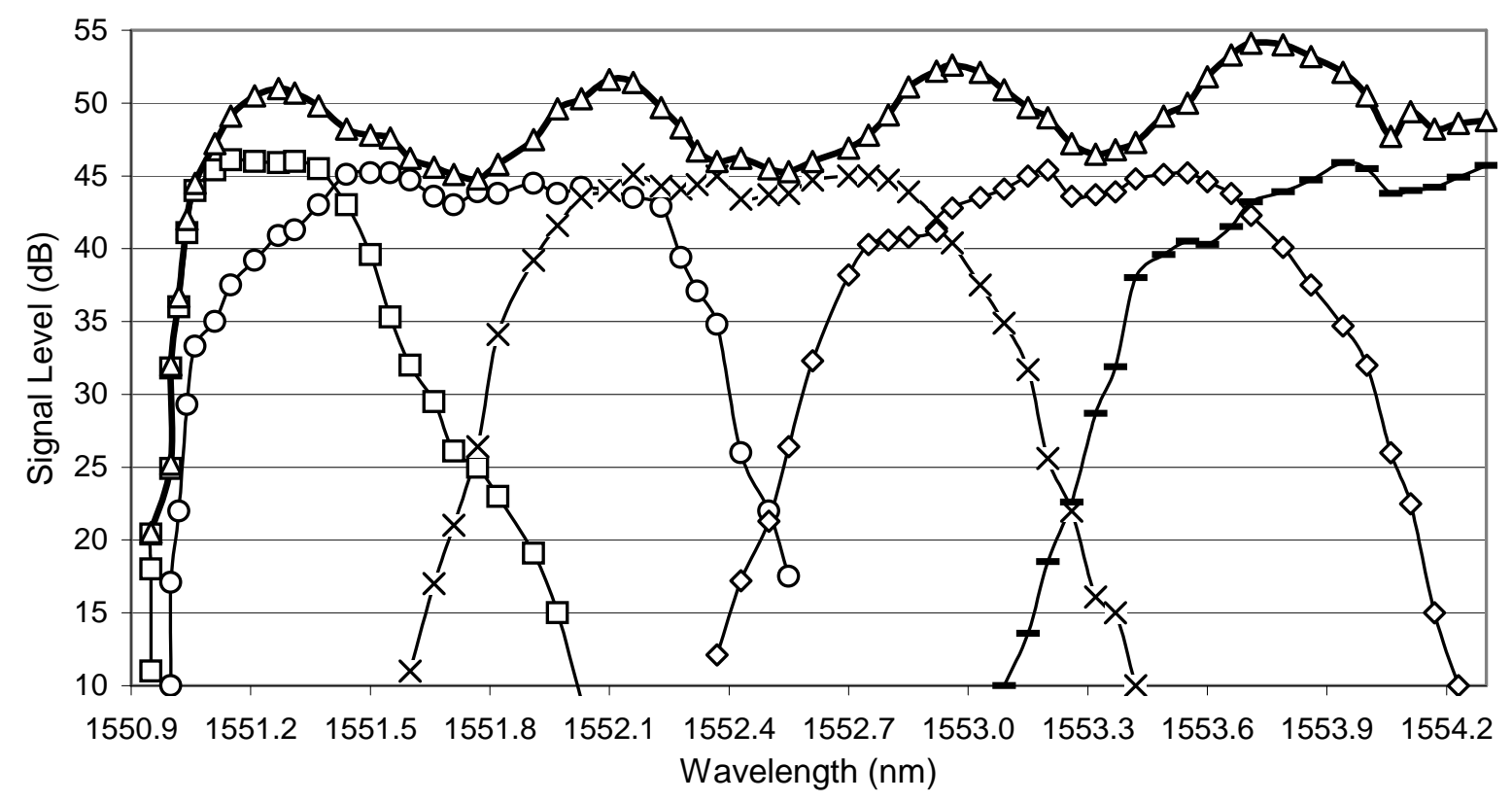

Fig. 11. Signal to noise ratio vs. wavelength for the third FBG stretched through channel 13 to channel 9 with a strain amplitude of $1.5 \mu \varepsilon$ at $30 \mathrm{~Hz}$. Channel 13 (squares), channel 12 (circles), channel 11 (crosses), channel 10 (diamonds), channel 9 (lines) and channels $13+12+11+10+9$ (triangles). 


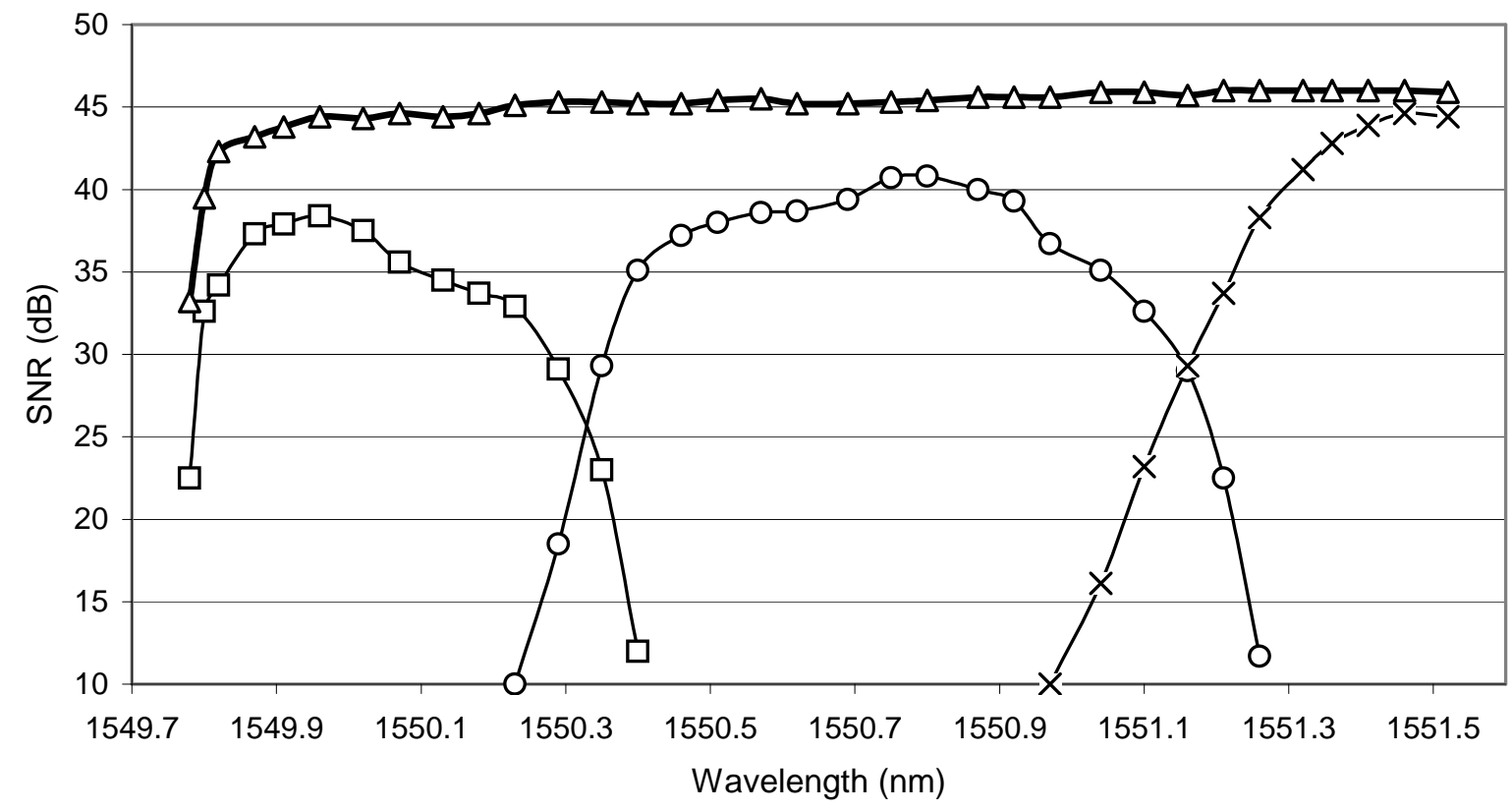

Fig. 12. Signal to noise ratio vs. wavelength for the double peaked FBG stretched through channel 14 to channel 12 with a strain amplitude of $1.5 \mu \varepsilon$ at $30 \mathrm{~Hz}$. Channel 14 (squares), channel 13 (circles), channel 12 (crosses) and channels $14+13+12$ (triangles). 